\title{
MicroRNA-4485 ameliorates severe influenza pneumonia via inhibition of the STAT3/PI3K/AKT signaling pathway
}

\author{
LONGFEI GUO*, QUANHONG WANG* and DONGQUAN ZHANG \\ Department of Critical Care Medicine, Gansu Provincial People's Hospital, Lanzhou, Gansu 730000, P.R. China
}

Received October 30, 2019; Accepted July 13, 2020

DOI: $10.3892 / \mathrm{ol} .2020 .12078$

\begin{abstract}
The present study aimed to explore the potential roles and mechanism of microRNA-4485 (miR-4485) in severe influenza pneumonia. miR-4485 expression was detected in patients with severe H1N1 pneumonia using quantitative PCR. Furthermore, the effects of aberrantly expressed miR-4485 on H1N1-infected A549 cells were investigated using Cell Counting Kit-8, terminal deoxynucleotidyl transferase dUTP nick end labeling, western blotting and (ELISA) assays. Furthermore, the regulatory relationships between miR-4485 and the STAT3-mediated PI3K/AKT/mTOR signaling pathway were explored using a luciferase reporter and rescue assay. MiR-4485 expression was downregulated following H1N1 infection and in patients with H1N1 pneumonia. In addition, miR-4485 alleviated H1N1-induced A549 cell injury by promoting cell viability and the production of cytokines, as well as reducing apoptosis in A549 cells. Furthermore, STAT3 was revealed to be a target gene of miR-4485. Additionally, STAT3 silencing reversed the protective effects of miR-4485 knockdown on H1N1-induced cell injury via inhibition of the $\mathrm{PI} 3 \mathrm{~K} / \mathrm{AKT} / \mathrm{mTOR}$ signaling pathway. In conclusion, miR-4485 inhibited H1N1-induced severe pneumonia in A549 cells by targeting STAT3 via the PI3K/AKT/mTOR signaling pathway.
\end{abstract}

\section{Introduction}

Influenza is an acute respiratory infectious disease caused by influenza virus. Humans often suffer from fever, cough, sore throat, headache, nasal discharge, fatigue and other symptoms following infection with influenza virus (1). In certain cases, severe complications induced by the influenza virus, such as pneumonia, respiratory failure, and multiple organ dysfunction,

Correspondence to: Dr Dongquan Zhang, Department of Critical Care Medicine, Gansu Provincial People's Hospital, 204 Donggang West Road, Lanzhou, Gansu 730000, P.R. China

E-mail: zhangdq62@126.com

*Contributed equally

Key words: H1N1 infection, microRNA-4485, PI3K/AKT/mTOR signaling pathway, severe pneumonia, STAT3 occur and may lead to death (2). In recent years, there have been frequent outbreaks of influenza viruses, and among these, the novel influenza A (H1N1) virus originating from the influenza pandemic in North America in 2009 has been identified as a leading cause of severe pneumonia (3). Therefore, H1N1 infection represents a critical threat to public health.

MicroRNAs (miRs/miRNAs) are endogenous single-stranded, non-coding RNA molecules with a length of 18-25 nucleotides, which are widely involved in the post-transcriptional regulation of target genes (4). Several miRNAs have been reported to be implicated in various biological and pathological processes, including pneumonia pathogenesis $(5,6)$. Previously, miR-4485 expression has been demonstrated to be downregulated in gastric cancer (7). Chen et al (8) identified miR-4485 as a potential biomarker of hepatitis B virus infection persistence. Furthermore, miR-4485 upregulation could suppress the tumorigenicity of breast cancer via regulation of mitochondrial functions (9). Recently, decreased serum expression levels of miR-4485 have been observed in severe pneumonia (10). However, the effects of miR-4485 on the development of severe H1N1 pneumonia are largely unknown.

In the present study, miR-4485 expression was detected in patients with severe H1N1 pneumonia. In addition, the effects of miR-4485 in H1N1-induced A549 cell injury were investigated by assessing cell viability, apoptosis and inflammation. Furthermore, the regulatory relationship between miR-4485 and STAT3 was explored, as well as the association between miR-4485 and the $\mathrm{PI} 3 \mathrm{~K} / \mathrm{AKT} / \mathrm{mTOR}$ signaling pathway. It was hypothesized that miR-4485 may promote cell viability, and inhibit cell apoptosis and the production of cytokines by targeting STAT3 and inactivating the $\mathrm{PI} 3 \mathrm{~K} / \mathrm{AKT} / \mathrm{mTOR}$ signaling pathway, thus alleviating H1N1-induced injury in A549 cells.

\section{Materials and methods}

Patients and controls. A series of 22 patients with confirmed influenza virus A (H1N1) pneumonia (18 males and 14 females; age range, 28-67 years; mean age, 45.7 years) and 18 age- and sex-matched healthy controls (10 males and 8 females; age range, 29-65 years; mean age, 44.6 years) were recruited at Gansu Provincial People's Hospital (Lanzhou, Gansu, China) between September and December, 2019. The present study was approved by the Ethics Committee of Gansu Provincial People's Hospital. Written informed consent was obtained from all subjects. 
A confirmed case of infection was defined as an acute lower respiratory tract illness with two or more of the following symptoms or signs: Cough, productive sputum, fever, chills, dyspnea, pleuritic chest pain, crackles and bronchial breathing plus an opacity or infiltrate seen on a chest radiography that was interpreted as pneumonia by the treating physicians, and a laboratory-confirmed $\mathrm{H} 1 \mathrm{~N} 1$ virus infection as confirmed by reverse transcription-quantitative PCR (RT-qPCR) or viral culture. A severe or critical case was defined as a case that met at least one of the following criteria on admission, according to the pH1N1 2009 Clinical guidelines (11) released by Chinese Ministry of Health (MOH): i) Respiratory failure; ii) Septic shock; iii) Multiple organ insufficiency; and iv) Other critical clinical conditions requiring intensive care. Hospitalized patients were excluded if they did not have pneumonia, or if they had been treated as outpatients or in emergency rooms, had a duration of hospitalization $<24 \mathrm{~h}$, or if there was an incomplete record of clinical outcome.

All patients in the present study had a body temperature $>100.4^{\circ} \mathrm{F}\left(>38^{\circ} \mathrm{C}\right)$, tachypnea and cough. Other common symptoms included diarrhea $(73 \%)$ and sore throat $(27 \%)$. A total of $5 \mathrm{ml}$ fasting peripheral blood samples was harvested from all subjects. Serum was obtained following centrifugation of blood samples at $4,000 \mathrm{x} \mathrm{g}$ at $4^{\circ} \mathrm{C}$ for $10 \mathrm{~min}$ and stored at $-80^{\circ} \mathrm{C}$.

Cell culture, transfection and treatment. Human pulmonary epithelial A549 cells and 293T cells, purchased from the American Type Culture Collection were cultured in DMEM supplemented with 10\% FBS (Gibco; Thermo Fisher Scientific, Inc.) and $100 \mu \mathrm{g} / \mathrm{ml}$ penicillin/streptomycin (Gibco; Thermo Fisher Scientific, Inc.) at $37^{\circ} \mathrm{C}$ with $5 \% \mathrm{CO}_{2}$.

When cell confluence reached $>70 \%$, the miR-4485 mimic $(50 \mathrm{nM}) /$ inhibitor $(150 \mathrm{nM})$, mimic/inhibitor negative control (mimic/inhibitor NC) $(50 \mathrm{nM} / 150 \mathrm{nM})$, small interfering (si)RNA against STAT3 or siRNA negative controls (si-NC) (100 nM) were transfected into cells using Lipofectamine 2000 (Invitrogen; Thermo Fisher Scientific, Inc.) according to the manufacturer's protocol. After $24 \mathrm{~h}$ of transfection, cells were infected with or without H1N1 at a MOI of 2 for another $24 \mathrm{~h}$. Primer sequences were as follows: miR-4485 mimic sense, 5'-UAACGGCCGCGGUACCCUAA-3' and mimic NC antisense, 5'-UGAUCCUCAGUUGUGUGUAA-3'; miR-4485 inhibitor 5'-UAACGGCCGCGGUACCCUAA-3'; si-STAT3: sense, 5'-GGACGACUUUGAUUUCAACtt-3'; antisense, 5'-GUUGAAAUCAAAGUCGUCCtg-3'. si-NC: sense, 5'-UUCUCCGAACGUGUCACGUTT-3'; antisense, 5'-ACG UGACACGUUCGGAGAATT-3'. A total of $48 \mathrm{~h}$ following transfection, the cells were collected for further experiments.

Luciferase reporter assay. The potential binding sites for miR-4485 in STAT3 were predicted using TargetScan Release 7.2 (http://www.targetscan.org/). Wild-type (WT)-STAT3-3' untranslated region (UTR)-pISo and MUT-STAT3-3'UTR-pISo luciferase reporter plasmids (50 ng) (Sangon Biotech Co., Ltd.) were co-transfected with either miR-4485 mimic or mimic NC into 293T cells using Lipofectamine 2000 (Invitrogen; Thermo Fisher Scientific, Inc.). After $48 \mathrm{~h}$ of transfection, the lysate was analyzed using a Dual-Glo luciferase assay system (Promega Corporation) and the luciferase activity was measured using a luminometer. Firefly luciferase activity was normalized to Renilla luciferase activity.

$R T$ - $q P C R$. Total RNA was isolated from peripheral blood samples or A549 cells using TRIzol reagent (Invitrogen; Thermo Fisher Scientific, Inc.), and purified to obtain the miRNA fraction using a mirVana miRNA isolation kit (Ambion; Thermo Fisher Scientific, Inc.) according to the manufacturer's protocol. Total RNA was reverse transcribed to cDNA using a PrimeScript RT reagent kit (Takara Biotechnology Co., Ltd.). A total of $1 \mu \mathrm{g}$ of total RNA was reversely transcribed using oligo(dT) primer at $42^{\circ} \mathrm{C}$ for $1 \mathrm{~h}$, and $2 \mu \mathrm{l}$ of the reverse transcription reaction mix was amplified by PCR with denaturation at $95^{\circ} \mathrm{C}$ for $2 \mathrm{~min}$, and 50 cycles at $95^{\circ} \mathrm{C}$ for $30 \mathrm{sec}, 55^{\circ} \mathrm{C}$ for $30 \mathrm{sec}$, and $72^{\circ} \mathrm{C}$ for $1 \mathrm{~min}$. The thermocycling conditions were as follows: Initial denaturation at $95^{\circ} \mathrm{C}$ for $30 \mathrm{sec}$, followed by 40 cycles of $95^{\circ} \mathrm{C}$ for $5 \mathrm{sec}$ and $60^{\circ} \mathrm{C}$ for $30 \mathrm{sec}$. The relative expression levels of miR-4485 were normalized to U6 using SYBR Premix Ex Taq (Takara Biotechnology Co., Ltd.) and calculated with the $2^{-\Delta \Delta \mathrm{Cq}}$ method (12). The primer sequences used in qPCR were as follows: U6, forward, 5'-GCTTCGGCA GCACATATACTAAAAT-3' and reverse, 5'-CGCTTCACG AATTTGCGTGTCAT-3'; and miR-4485 Forward, 5'-AGU AACGGCCGCGGUAC-3' and reverse, 5'-TCCAGTTTTTTT TTTTTTTTTAGG-3'.

Cell Counting Kit-8 (CCK-8) assay. The CCK-8 reagent kit (Beyotime Institute of Biotechnology) was used to analyze the viability rates of A549 cells according to the manufacturer's protocol. At 24, 48 and $72 \mathrm{~h}$ after transfection, cells were treated with $10 \mu \mathrm{l}$ CCK-8 solution for an additional $2 \mathrm{~h}$. The optical density was measured at $450 \mathrm{~nm}$.

Apoptosis detection. The TUNEL reaction was performed according to the manufacturer's protocols (Roche Applied Science). After rinsing twice with PBS, A549 cells were fixed with $4 \%$ paraformaldehyde for $15 \mathrm{~min}$ at room temperature, permeabilized in $0.25 \%$ Triton X-100 on ice for $20 \mathrm{~min}$, and incubated in terminal dexynucleotidyl transferase reaction cocktail for $45 \mathrm{~min}$ at $37^{\circ} \mathrm{C}$. Cell nuclei were visualized by DAPI staining $(0.3 \mu \mathrm{g} / \mathrm{ml}$; Sigma-Aldrich; Merck KGaA) at $4^{\circ} \mathrm{C}$ for $10 \mathrm{~min}$. The slides were mounted using DAPI-Fluoromount-GTM, covered with glass slides, and four fields of view were examined in using a scanning flourescence microscope.

Western blotting. A549 cells were harvested and lysed using RIPA buffer (Sigma-Aldrich; Merck KGaA). Protein concentrations were determined using the BCA protein assay kit (Thermo Fisher Scientific, Inc.). In total, $30 \mu \mathrm{g}$ proteins were loaded on a $10 \%$ gel, resolved using SDS-PAGE and transferred onto a nitrocellulose membrane. The membrane was blocked in 5\% skimmed milk at room temperature for $30 \mathrm{~min}$. Western blotting was performed using antibodies against Bax (\#5023), Bcl-2 (\#3498), STAT3 (\#12640), phosphorylated (p)-STAT3 (\#9145), PI3K (\#4249), p-PI3K (\#17366), AKT (\#4685), p-AKT (\#4060), mTOR (\#2983) and p-mTOR (\#5536) (all 1:1,000; Cell Signaling Technology, Inc.) at $4^{\circ} \mathrm{C}$ overnight. Membranes were incubated with HRP-coupled IgG antibody (\#7074; 1:1,000; 
$\mathrm{A}$

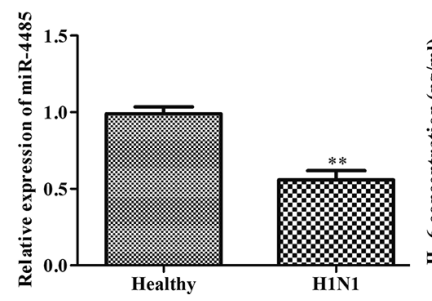

B

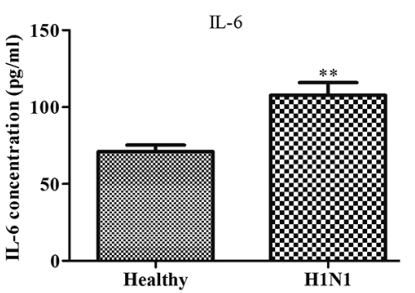

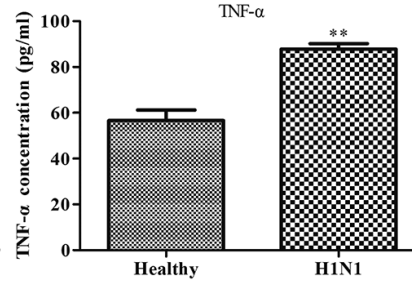

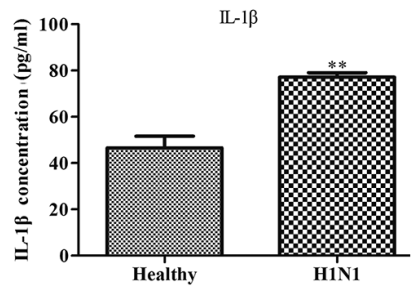

Figure 1. miR-4485 expression is downregulated in patients with severe H1N1 pneumonia. (A) Serum expression levels of miR-4485 were determined using reverse transcription-quantitative PCR, and (B) IL-6, TNF- $\alpha$ and IL-1 $\beta$ levels were determined using ELISA in patients with severe H1N1 pneumonia (n=22) and healthy controls $(\mathrm{n}=18)$. The experiments were performed three times and data are presented as the mean $\pm \mathrm{SD}$. ${ }^{* *} \mathrm{P}<0.01 \mathrm{vs}$. the healthy controls. miR- 4485 , microRNA- 4485 .

Cell Signaling Technology, Inc.) at room temperature for $1 \mathrm{~h}$. Densitometry analysis of the bands was performed using the ImageJ software (version 1.52; National Institutes of Health). GAPDH was used as the loading control.

ELISA. Serum samples and supernatants from cultured A549 cells were collected and the concentrations of pro-inflammatory cytokines IL- 6 , TNF- $\alpha$ and IL- $1 \beta$ were measured using Human IL-6 Quantikine ELISA Kit (cat. no. D6050), Human TNF-alpha Quantikine ELISA Kit (cat. no. DTA00D) and Human IL-1 $\beta /$ IL-1F2 Quantikine ELISA Kit (cat. no. DLB50) (R\&D Systems, Inc.) according to the manufacturer's protocols.

Statistical analysis. Statistical analysis was performed using SPSS 19.0 statistical software (IBM Corp.). Data are presented as the mean \pm SD from at least three independent experiments. Differences among groups were compared using Student's t-test or one-way ANOVA with Tukey's post hoc test. $\mathrm{P}<0.05$ was considered to indicate a statistically significant difference.

\section{Results}

miR-4485 expression is downregulated in patients with severe H1N1 pneumonia. As shown in Fig. 1A, serum miR-4485 expression was significantly downregulated in patients with severe H1N1 pneumonia compared with in healthy subjects. Furthermore, ELISA results demonstrated that patients with severe H1N1 pneumonia had higher serum levels of IL-6, TNF- $\alpha$ and IL-1 $\beta$ compared with the healthy controls (Fig. 1B).

Overexpression of miR-4485 improves H1N1-induced A549 cell injury. To determine whether miR-4485 was involved in the development of severe H1N1 pneumonia, A549 cells were infected with H1N1 to establish a cell model of pneumonia. As shown in Fig. 2A, H1N1 infection decreased miR-4485 expression. To investigate the effects of miR-4485 on H1N1 infection-induced A549 cell injury, A549 cells were transfected with miR-4485 mimic to overexpress miR-4485 and successful transfection was confirmed by RT-qPCR (Fig. 2B). Furthermore, the present data demonstrated that overexpression of miR-4485 markedly ameliorated H1N1-induced A549 cell injury by promoting cell viability (Fig. 2C), inhibiting apoptosis (Fig. 2D), reversing H1N1-induced expression changes of apoptosis-related proteins (Fig. 2E), and suppressing the secretion of IL-6, TNF- $\alpha$ and IL-1 $\beta$ (Fig. 2 F).
STAT3 is a direct target gene of $m i R-4485$. It has been demonstrated that the STAT3-mediated PI3K/AKT/mTOR signaling pathway may serve a key role in the process of lung injury (13). In the present study, H1N1 infection resulted in increased protein phosphorylation levels of PI3K, AKT and mTOR, as demonstrated by an enhanced ratio of $\mathrm{p}-\mathrm{PI} 3 \mathrm{~K} / \mathrm{PI} 3 \mathrm{~K}$, p-AKT/AKT and p-mTOR/mTOR (Fig. 3A). According to the predicted results of TargetScan, STAT3 was identified as a potential target of miR-4485. To confirm the predicted results, a luciferase reporter assay was performed, and the results revealed that the relative luciferase activities of STAT3 3'UTR WT were significantly decreased in the miR-4485 mimic group compared with in the mimic NC group (Fig. 3B). RT-qPCR analysis confirmed the knockdown of miR-4485 expression in A549 cells following transfection with miR-4485 inhibitor (Fig. 3C). Furthermore, the phosphorylated STAT3, $\mathrm{PI} 3 \mathrm{~K}, \mathrm{AKT}$ and $\mathrm{mTOR} /$ total protein ratios were significantly reduced in the miR-4485 mimic group compared with in the mimic NC group, whereas they were markedly increased in the miR-4485 inhibitor group compared with in the inhibitor NC group (Fig. 3D).

STAT3 knockdown alleviates the effects of miR-4485 inhibition on H1N1-induced A549 cell injury. To explore the regulatory relationship between miR-4485 and STAT3, STAT3 expression was knocked down by transfection with si-STAT3 in A549 cells following miR-4485 downregulation by transfection with miR-4485 inhibitor. As shown in Fig. 4A, si-STAT3 transfection decreased the total and phosphorylated protein levels of STAT3. Furthermore, the suppression of miR-4485 expression deteriorated H1N1-induced PI3K/AKT/mTOR phosphorylation (Fig. 4B) with no change in STAT3 expression, inhibited cell viability (Fig. 4C), promoted apoptosis (Fig. 4D) via upregulation of Bax expression and downregulation of the expression levels of $\mathrm{Bcl}-2$ (Fig. 4E), and increased production of cytokines (Fig. 4F), and these effects were partly reversed by si-STAT3 transfection.

\section{Discussion}

Maintaining epithelial integrity in the lung is critical in fighting infection and allowing optimal gas exchange. Influenza viruses disrupt epithelial homeostasis as they directly target epithelial cells in the lung mucosa (14). Severe infection causes excessive epithelial injury, fluid buildup in the lung parenchyma and increased susceptibility to secondary bacterial infections (15). 

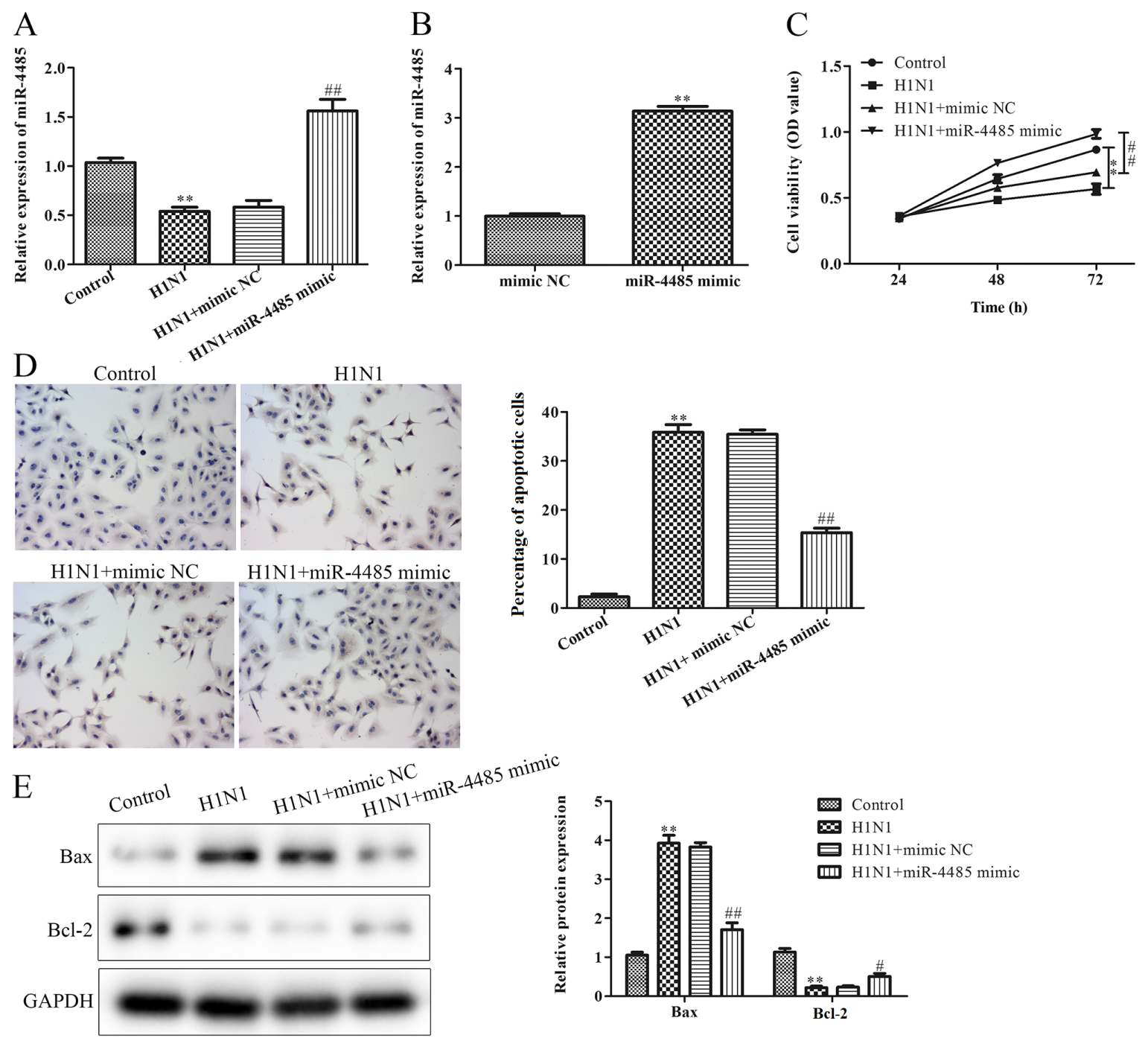

$-4485 \mathrm{mimic}$
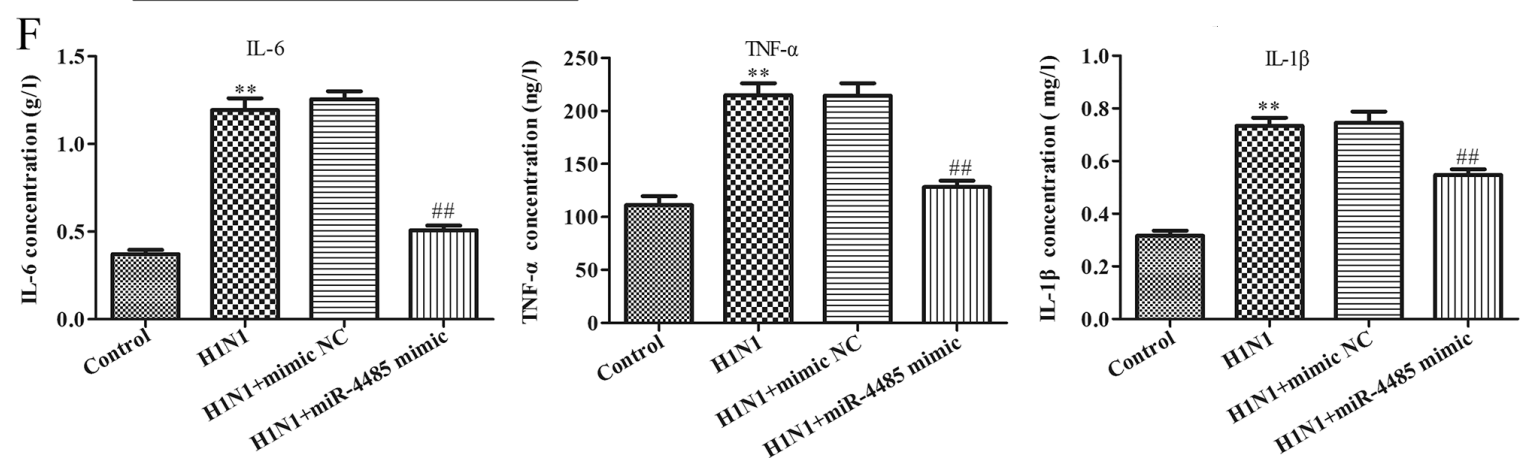

Figure 2. Overexpression of miR-4485 improves H1N1-induced A549 cell injury. (A) Expression levels of miR-4485 in A549 cells infected with or without H1N1 after transfection with miR-4485 mimic or mimic NC. (B) Reverse transcription-quantitative PCR demonstrated miR-4485 overexpression in A549 cells following transfection with miR-4485 mimic. (C) Cell Counting Kit-8 cell viability assay, (D) TUNEL apoptosis detection (magnification x200), (E) relative protein expression of Bax and Bcl-2, and (F) production of IL-6, TNF- $\alpha$ and IL-1 $\beta$ in A549 cells infected with or without H1N1 following transfection with miR-4485 mimic or mimic NC. The experiments were performed three times and the data are presented as the mean $\pm \mathrm{SD}$. ${ }^{* *} \mathrm{P}<0.01 \mathrm{vs}$. the control group; ${ }^{\#} \mathrm{P}<0.05,{ }^{\# /} \mathrm{P}<0.01$ vs. H1N1 + mimic NC group. miR-4485, microRNA-4485; NC, negative control; OD, optical density.

Endothelial cells are considered to be the central regulators of cytokine storm in the respiratory system during influenza virus infection (16). Previous studies have revealed that infection with H1N1 triggers the release of proinflammatory cytokines in human lung epithelial A549 cells $(17,18)$. Understanding how epithelial cells respond to influenza infection is important for optimizing immune-based strategies to reduce the burden of infection.
Several lines of evidence suggest that some miRNAs function as crucial regulators in the pathological process of influenza pneumonia. For example, Zhang et al (19) reported that miR-29c promotes H1N1 infection-induced inflammatory and antiviral signaling in A549 cells. Podsiad et al (20) revealed that miR-155 increases mortality and impairs bacterial clearance in post-influenza pneumonia via negative regulation of the IL-23/IL-17 signaling pathway. 

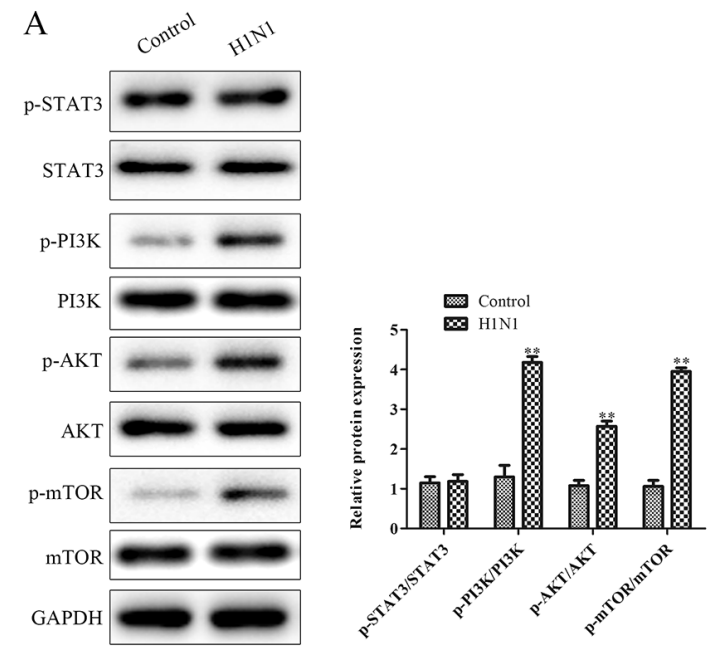

B
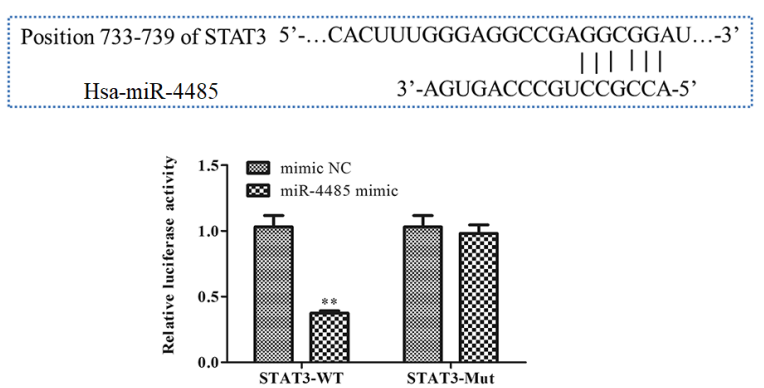
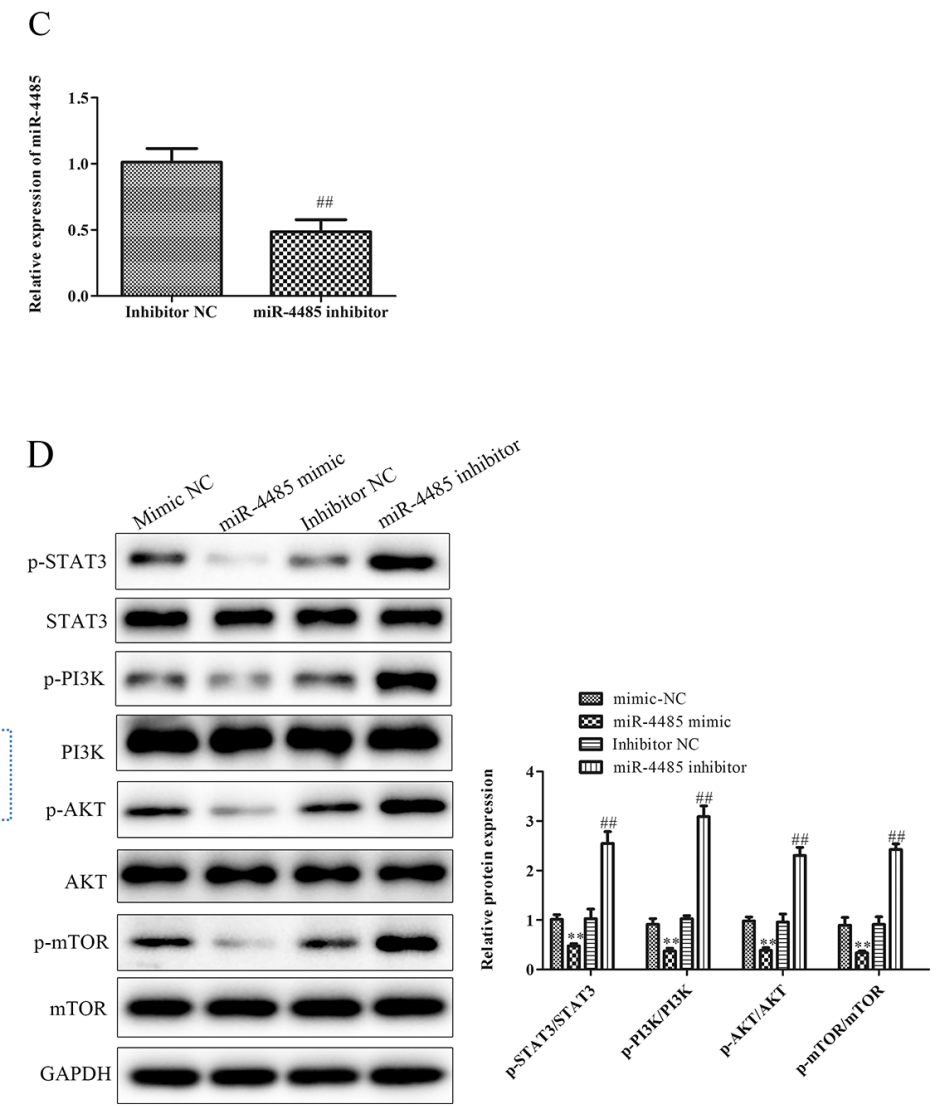

Figure 3.STAT3 is a direct target gene of miR-4485.(A) Phosphorylated and total protein levels of STAT3,PI3K, AKT and mTOR and the ratio of p-STAT3/STAT3, p-PI3K/PI3K, p-AKT/AKT and p-mTOR/mTOR in A549 cells infected with or without H1N1. (B) Predicted binding sequences of STAT3 and miR-4485. A luciferase reporter assay demonstrated that STAT3 3' untranslated region-WT was targeted by miR-4485 in $293 \mathrm{~T}$ cells. (C) Reverse transcription-quantitative PCR confirmation of miR-4485 knockdown in A549 cells following transfection with miR-4485 inhibitor. (D) Phosphorylated and total protein levels of STAT3, PI3K, AKT and mTOR and the ratio of p-STAT3/STAT3, p-PI3K/PI3K, p-AKT/AKT and p-mTOR/mTOR in A549 cells transfected with miR-4485 mimic/inhibitor or mimic/inhibitor NC. The experiments were performed three times and the data are presented as the mean $\pm \mathrm{SD}$. ${ }^{* *} \mathrm{P}<0.01 \mathrm{vs}$. control or mimic NC group; ${ }^{\# /} \mathrm{P}<0.01$ vs. inhibitor NC group. miR-4485, microRNA-4485; Mut, mutant; NC, negative control; $p-$, phosphorylated; WT, wild type.

Liu et al (21) demonstrated that inhibition of miR-200c-3p ameliorates lung injury induced by $\mathrm{H} 5 \mathrm{~N} 1$ virus infection by targeting angiotensin-converting enzyme 2 . To the best of our knowledge, the present study was the first to demonstrate the downregulation of miR-4485 expression in patients with severe H1N1 pneumonia. Results from in vitro experiments demonstrated that H1N1 infection markedly induced cell injury by inhibiting cell viability, inducing cell apoptosis and enhancing the production of cytokines. Additionally, H1N1 inoculation resulted in decreased expression levels of miR-4485 in A549 cells. In addition, overexpression of miR-4485 alleviated H1N1-induced cell injury.

There is emerging evidence that miRNAs serve crucial roles in several biological processes by regulating their target genes. In the present study, STAT3, an inflammation-related transcription factor, was identified as a direct target gene of miR-4485. Increasing evidence has suggested an association between STAT3 and the development of pneumonia. For instance, STAT3 inhibition rescues mice from pulmonary Staphylococcus aureus infections by targeting the antimicrobial protein regenerating islet-derived $3 \gamma$ (22). Traber et al (23) demonstrated that STAT3 is required for neutrophil and macrophage recruitment during pneumonia by inducing chemokine $\mathrm{C}-\mathrm{X}-\mathrm{C}$ motif ligand 5 expression. Additionally, STAT3 inhibition could suppress lipopolysaccharide-induced macrophage and inflammatory cell infiltration in lung and bronchoalveolar lavage fluid (24). In the present study, STAT3 was demonstrated to be a direct target gene of miR-4485. In addition, knockdown of STAT3 could reverse the effects of miR-4485 downregulation on H1N1-induced cell injury. Overall, these findings suggest that miR-4485 may serve a crucial role in H1N1-induced severe pneumonia by inhibiting STAT3 expression. However, the functional regulatory mechanisms of STAT3 and miR-4485 remain to be elucidated.

Increasing evidence has revealed the critical roles of the PI3K/AKT/mTOR signaling pathway in the host response against pneumovirus infections. For instance, Klebsiella pneumoniae induces autophagy and oxidative stress in Caenorhabditis elegans through inhibition of the PI3K/AKT/mTOR signaling pathway (25). Streptococcus pneumonia induces autophagy in A549 cells via a PI3K/AKT/mTOR-dependent mechanism (26). Yang et al (27) demonstrated that inhibition of $\mathrm{PI} 3 \mathrm{~K} / \mathrm{AKT} / \mathrm{mTOR}$ decreases the reinfection of Streptococcus pneumonia following influenza A virus infection in community-acquired pneumonia. Therefore, the present study aimed to explore whether the effects of miR-4485 on H1N1-induced severe pneumonia could be mediated via the PI3K/AKT/mTOR signaling pathway. In the present study, the PI3K/AKT/mTOR signaling pathway 
A
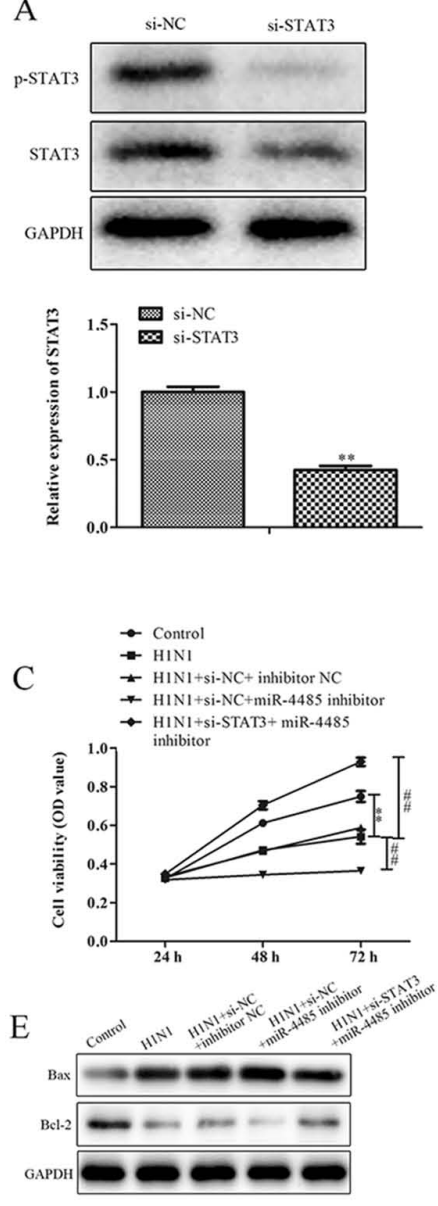
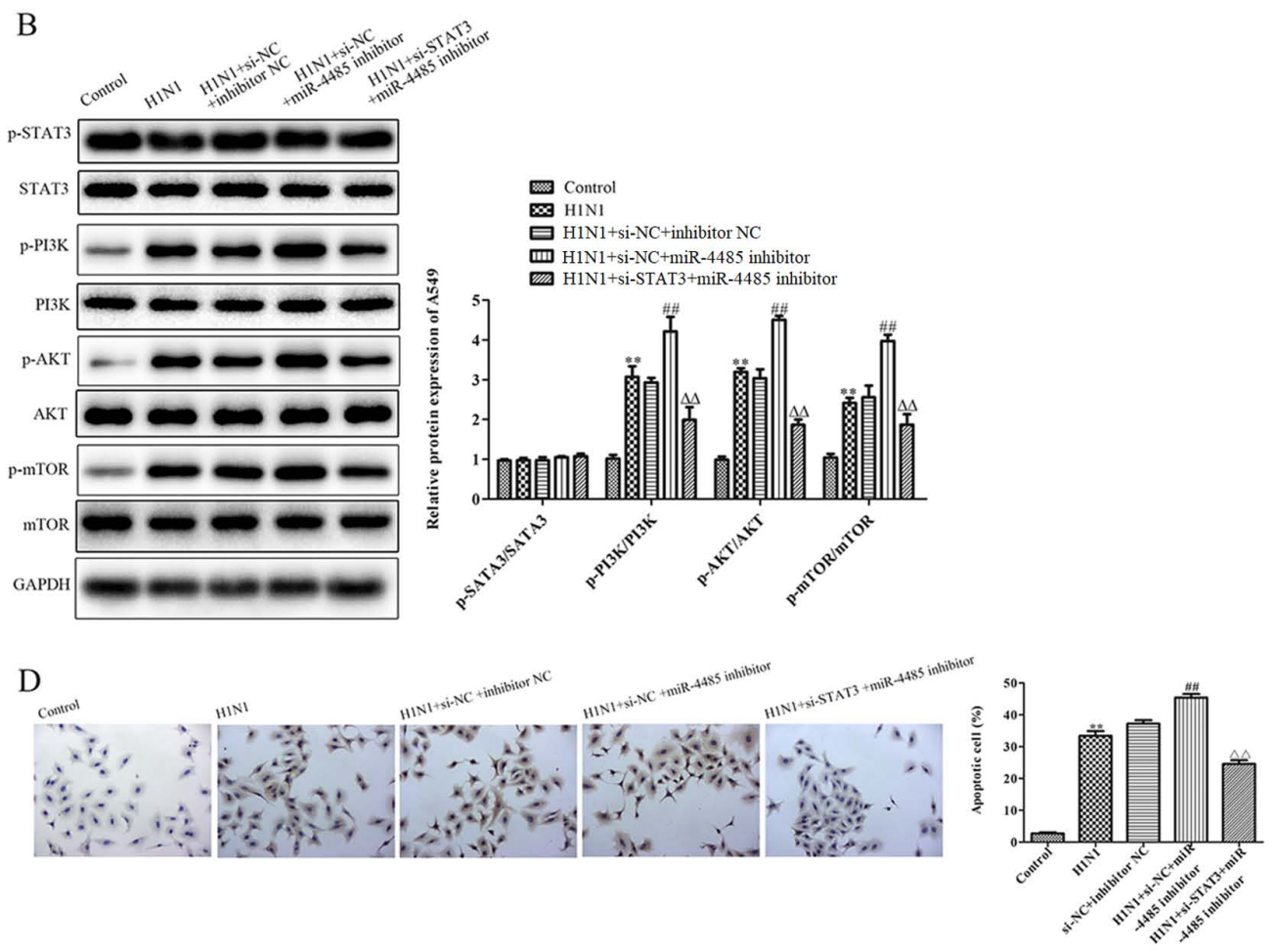
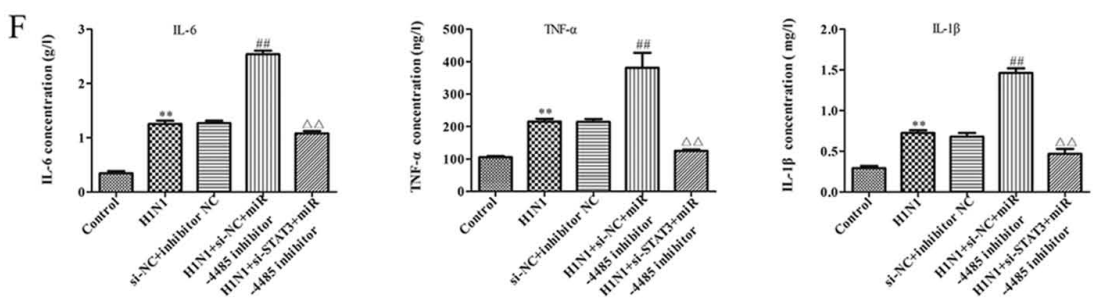

Figure 4. STAT3 knockdown alleviates the effects of miR-4485 inhibition on H1N1-induced A549 cell injury. (A) Western blotting demonstrated STAT3 knockdown in A549 cells following transfection with si-STAT3. (B) Phosphorylated and total protein levels of STAT3, PI3K, AKT and mTOR and the ratio of p-STAT3/STAT3, p-PI3K/PI3K, p-AKT/AKT and p-mTOR/mTOR in A549 cells. (C) Cell Counting Kit-8 cell viability assay, (D) TUNEL apoptosis detection (magnification x200), (E) protein expression levels of Bax and Bcl-2, and (F) production of IL-6, TNF- $\alpha$ and IL-1 $\beta$ in A549 cells infected with or without H1N1 following co-transfection with miR-4485 inhibitor and/or si-STAT3. The experiments were performed three times and the data are presented as the mean $\pm \mathrm{SD} .{ }^{* *} \mathrm{P}<0.05$ vs. control group; ${ }^{\# \#} \mathrm{P}<0.05$ vs. H1N1 + si-NC + inhibitor $\mathrm{NC}$ group; ${ }^{\Delta \Delta} \mathrm{P}<0.01$ vs. H1N1 + si-NC + miR-4485 inhibitor group. miR-4485, microRNA-4485; Mut, mutant; NC, negative control; OD, optical density; p-, phosphorylated; si, small interfering RNA.

was activated following inhibition of miR-4485, and was markedly inhibited by STAT3 silencing, suggesting the regulatory functions of miR-4485 on the STAT3-mediated $\mathrm{PI} 3 \mathrm{~K} / \mathrm{AKT} / \mathrm{mTOR}$ pathway. Therefore, it was speculated that miR-4485 may target STAT3 to regulate the activation of the PI3K/AKT/mTOR signaling pathway, thus mediating the development of severe H1N1 pneumonia.

In summary, the present study revealed that miR-4485 expression was downregulated in patients with severe H1N1 pneumonia, and that downregulation of miR-4485 may promote H1N1 infection-induced A549 cell injury by targeting STAT3 via the activation of the PI3K/AKT/mTOR signaling pathway. These findings will provide a potential therapeutic target for the treatment of severe H1N1 pneumonia.

\section{Acknowledgements}

Not applicable.

\section{Funding}

The study was funded by the Key Subject Project of Gansu Provincial Hospital (grant. no. 20GSSY1-14).

\section{Availability of data and materials}

The datasets used and/or analyzed during the current study are available from the corresponding author on reasonable request. 


\section{Authors' contributions}

LG and QW conducted the majority of the experiments, wrote the manuscript and analyzed the data. DZ designed the study and revised the manuscript. All authors read and approved the final manuscript.

\section{Ethics approval and consent to participate}

All experimental procedures were approved by the Human Ethics Committee of Gansu Provincial People's Hospital (approval no. GSYYR20180912).

\section{Patient consent for publication}

Not applicable.

\section{Competing interests}

The authors declare that they have no competing interests.

\section{References}

1. Gurav YK, Chadha MS, Tandale BV, Potdar VA, Pawar SD, Shil P, Deoshatwar AR, Aarthy R and Bhushan A: Influenza $\mathrm{A}(\mathrm{H} 1 \mathrm{~N} 1) \mathrm{pdm} 09$ outbreak detected in inter-seasonal months during the surveillance of influenza-like illness in Pune, India, 2012-2015. Epidemiol Infect 145: 1898-1909, 2017.

2. Peteranderl C, Herold S and Schmoldt C: Human influenza virus infections. Semin Respir Crit Care Med 37: 487-500, 2016.

3. Li H, Yang SG, Gu L, Zhang Y, Yan XX, Liang ZA, Zhang W, Jia HY, Chen W, Liu M, et al: National influenza apdm09 clinical investigation group of, effect of low-to-moderate-dose corticosteroids on mortality of hospitalized adolescents and adults with influenza $A(\mathrm{H} 1 \mathrm{~N} 1)$ pdm09 viral pneumonia. Influenza Other Respir Viruses 11: 345-354, 2017.

4. Kalogianni DP, Kalligosfyri PM, Kyriakou IK and Christopoulos TK: Advances in microRNA analysis. Anal Bioanal Chem 410: 695-713, 2018.

5. Hoffmann J, Machado D, Terrier O, Pouzol S, Messaoudi M, Basualdo W, Espínola EE, Guillen RM, Rosa-Calatrava M, Picot V, et al: Paranhos-baccala, viral and bacterial co-infection in severe pneumonia triggers innate immune responses and specifically enhances IP-10: A translational study. Sci Rep 6: 38532,2016

6. Lin J, Wang Y, Zou YQ, Chen X, Huang B, Liu J, Xu YM, Li J, Zhang J, Yang WM, et al: Differential miRNA expression in pleural effusions derived from extracellular vesicles of patients with lung cancer, pulmonary tuberculosis, or pneumonia. Tumour Biol 14: 1007, 2016.

7. Yang B, Jing C, Wang J, Guo X, Chen Y, Xu R, Peng L, Liu J and Li L: Identification of microRNAs associated with lymphangiogenesis in human gastric cancer. Clin Transl Oncol 16: 374-379, 2014.

8. Chen J, Wu X, Cai B, Su Z, Li L, An Y and Wang L: Circulating microRNAs as potential biomarkers of HBV infection persistence. Infect Genet Evol 54: 152-157, 2017.

9. Sripada L, Singh K, Lipatova AV, Singh A, Prajapati P, Tomar D, Bhatelia K, Roy M, Singh R, Godbole MM, et al: Hsa-MiR-4485 regulates mitochondrial functions and inhibits the tumorigenicity of breast cancer cells. J Mol Med (Berl) 95: 641-651, 2017.

10. Huang S, Feng C, Zhai YZ, Zhou X, Li B, Wang LL, Chen W, Lv FQ and Li TS: Identification of miRNA biomarkers of pneumonia using RNA-sequencing and bioinformatics analysis. Exp Ther Med 13: 1235-1244, 2017.

11. Yang SG, Cao B, Liang LR, Li XL, Xiao YH, Cao ZX, Jia HY, $\mathrm{Yu} \mathrm{HJ}, \mathrm{Xu} \mathrm{Z}, \mathrm{Gu} \mathrm{L}$, et al: Antiviral therapy and outcomes of patients with pneumonia caused by influenza a pandemic (H1N1) virus. PLoS One 7: e29652, 2012.
12. Livak KJ and Schmittgen TD: Analysis of relative gene expression data using real-time quantitative PCR and the 2(-Delta Delta C(T)) method. Methods 25: 402-408, 2001.

13. Zhao YR, Wang D, Liu Y, Shan L and Zhou JL: The PI3K/Akt, p38MAPK, and JAK2/STAT3 signaling pathways mediate the protection of $\mathrm{SO} 2$ against acute lung injury induced by limb ischemia/reperfusion in rats. J Physiol Sci 66: 229-239, 2016.

14. Traylor ZP, Aeffner F and Davis IC: Influenza A H1N1 induces declines in alveolar gas exchange in mice consistent with rapid post-infection progression from acute lung injury to ARDS. Influenza Other Respir Viruses 7: 472-479, 2013.

15. Siemens N, Oehmcke-Hecht S, Mettenleiter TC, Kreikemeyer B, Valentin-Weigand P and Hammerschmidt S: Port d'Entree for respiratory infections-does the influenza a virus pave the way for bacteria? Front Microbiol 8: 2602, 2017.

16. Wang L, Jiang H, Shen SM, Wen CX, Xing Z and Shi Y: Inhibition of autophagy and chemokine induction by sphingosine 1-phosphate receptor 1 through NF- $\mathrm{BB}$ signaling in human pulmonary endothelial cells infected with influenza a viruses. PLoS One 13: e0205344.

17. Teijaro JR, Walsh KB, Cahalan S, Fremgen DM, Roberts E, Scott F, Martinborough E, Peach R, Oldstone MB and Rosen H: Endothelial cells are central orchestrators of cytokine amplification during influenza virus infection. Cell 146: 980-991, 2011

18. Guo X, Zhu Z, Zhang W, Meng X, Zhu Y, Han P, Zhou X, $\mathrm{Hu}$ Y and Wang R: Nuclear translocation of HIF-1 $\alpha$ induced by influenza $A(H 1 N 1)$ infection is critical to the production of proinflammatory cytokines. Emerg Microbes Infect 6: e39, 2017.

19. Zhang X, Dong C, Sun X, Li Z, Zhang M, Guan Z and Duan M: Induction of the cellular miR-29c by influenza virus inhibits the innate immune response through protection of A20 mrna. Biochem Biophys Res Commun 450: 755-761, 2014.

20. Podsiad A, Standiford TJ, Ballinger MN, Eakin R, Park P, Kunkel SL, Moore BB and Bhan U: MicroRNA-155 regulates host immune response to postviral bacterial pneumonia via IL-23/IL-17 pathway. Am J Physiol Lung Cell Mol Physiol 310: L465-L475, 2016.

21. Liu Q, Du J, Yu X, Xu J, Huang F, Li X, Zhang C, Li X, Chang J, Shang D, et al: MiRNA-200c-3p is crucial in acute respiratory distress syndrome. Cell Discov 3: 17021, 2017.

22. Choi SM, McAleer JP, Zheng M, Pociask DA, Kaplan MH, Qin S, Reinhart TA and Kolls JK: Innate stat3-mediated induction of the antimicrobial protein Reg3g is required for host defense against MRSA pneumonia. J Exp Med 210: 551-561, 2013.

23. Traber KE, Hilliard KL, Allen E, Wasserman GA, Yamamoto K, Jones MR, Mizgerd JP and Quinton LJ: Induction of STAT3-dependent CXCL5 expression and neutrophil recruitment by oncostatin-M during pneumonia. Am J Respir Cell Mol Biol 53: 479-488, 2015.

24. Zhao J, Yu H, Liu Y, Gibson SA, Yan Z, Xu X, Gaggar A, Li PK, Li C, Wei S, et al: Protective effect of suppressing STAT3 activity in LPS-induced acute lung injury. Am J Physiol Lung Cell Mol Physiol 311: L868-L880, 2016.

25. Kamaladevi A and Balamurugan K: Global proteomics revealed klebsiella pneumoniae induced autophagy and oxidative stress in caenorhabditis elegans by inhibiting PI3K/AKT/mTOR pathway during infection. Front Cell Infect Microbiol 7: 393, 2017.

26. Li P, Shi J, He Q, Hu Q, Wang YY, Zhang LJ, Chan WT and Chen WX: Streptococcus pneumoniae induces autophagy through the inhibition of the PI3K-I/Akt/mTOR pathway and ROS hypergeneration in A549 cells. PLoS One 10: e0122753, 2015.

27. Yang Z, Zou X, Feng P, Zhan H, Xiong D and Lang J: Inhibition of the PI3K/AKT signaling pathway or overexpression of beclin1 blocks reinfection of streptococcus pneumoniae after infection of influenza a virus in severe community-acquired pneumonia. Inflammation 42: 1741-1753, 2019.

This work is licensed under a Creative Commons Attribution-NonCommercial-NoDerivatives 4.0 International (CC BY-NC-ND 4.0) License. 